OPEN ACCESS

Edited by:

Eve Mary Dorothy Smith,

University of Liverpool,

United Kingdom

Reviewed by:

Toni Hospach,

Klinikum Stuttgart, Germany

Andrew R. Gennery,

Newcastle University, United Kingdom

*Correspondence:

Marco Cazzaniga

marco.cazzaniga1@unimi.it

Specialty section:

This article was submitted to

Pediatric Immunology,

a section of the journal

Frontiers in Pediatrics

Received: 29 April 2020

Accepted: 10 June 2020

Published: 03 July 2020

Citation:

Cazzaniga M, Baselli LA, Cimaz R,

Guez SS, Pinzani $R$ and

Dellepiane RM (2020) SARS-COV-2

Infection and Kawasaki Disease: Case

Report of a Hitherto Unrecognized

Association. Front. Pediatr. 8:398

doi: 10.3389/fped.2020.00398

\section{SARS-COV-2 Infection and Kawasaki Disease: Case Report of a Hitherto Unrecognized Association}

\author{
Marco Cazzaniga ${ }^{1 *}$, Lucia Augusta Baselli², Rolando Cimaz ${ }^{3}$, Sophie Suzanne Guez ${ }^{4}$, \\ Raffaella Pinzani ${ }^{4}$ and Rosa Maria Dellepiane ${ }^{2}$ \\ ${ }^{1}$ Pediatric Intermediate Care Unit, Fondazione IRCCS Ca' Granda Ospedale Maggiore Policlinico, University of Milan, Milan, \\ Italy, ${ }^{2}$ Pediatric Intermediate Care Unit, Fondazione IRCCS Ca' Granda Ospedale Maggiore Policlinico, Milan, Italy, \\ ${ }^{3}$ Department of Clinical Sciences and Community Health, Research Center for Adult and Pediatric Rheumatic Diseases, \\ University of Milan, Milan, Italy, ${ }^{4}$ Pediatric Highly Intensive Care Unit, Fondazione IRCCS Ca' Granda Ospedale Maggiore \\ Policlinico, Milan, Italy
}

Coronavirus-associated disease (COVID-19) was firstly reported at the end of 2019. Generally, COVID-19 seems to be a less severe disease in children than in adults. According to the current literature, children account approximately for $2 \%$ of diagnosed COVID-19 cases. Northern Italy is one of the geographical areas mainly affected by the ongoing COVID-19 pandemic. We describe a pediatric patient diagnosed and treated for atypical/incomplete Kawasaki Disease (KD) complicated with paralytic ileus, who also resulted positive for SARS-COV-2.

Keywords: Kawasaki disease, SARS-COV-2, children, paralytic ileus, cytokine storm

\section{INTRODUCTION}

Covid-19 infection is currently the most important health problem worldwide. According to the current literature, children account approximately for $2 \%$ of diagnosed COVID-19 cases (1-3). Even if most of medical efforts are aimed to fight this disease, physicians still have to care for other acute and chronic disorders. Kawasaki disease is not an exception, and there may be a risk that patients are not recognized early since patients may not seek medical care as usual for fear of getting infected (4). The association of Kawasaki disease and COVID-19 infection has to our knowledge been reported only once (5), we report a second case from Italy, currently the third most affected country in the world with regard to number of proven SARS-COV-2 infections.

\section{CASE REPORT}

We report the case of a 6-year-old, full term, previously healthy boy. In March 2020 he was seen by his pediatrician for fever lasting for 3 days, sore throat and asthenia, and antibiotic therapy with amoxicillin + clavulanic acid was prescribed. High fever persisted with vomiting and diarrhea appearing on day 4 and 5. On day 6 from onset of fever, erythematous rash in the back and hands, labial and conjunctival hyperemia appeared, and the child was admitted to a regional hospital. Laboratory tests showed white blood cells count of $10.300 / \mathrm{mm}^{3}$ (neutrophils $88.6 \%$, lymphocytes 7.1\%), $\mathrm{Hb} 11.3 \mathrm{~g} / \mathrm{dl}$, platelets $1,49,000 / \mathrm{mm}^{3}$, elevated liver enzymes (AST 73 U/L, ALT $189 \mathrm{U} / \mathrm{L}$, GGT $128 \mathrm{U} / \mathrm{L}$ ), C-reactive protein $13 \mathrm{mg} / \mathrm{dL}$ ( $\mathrm{nv}<5$ ), fibrinogen $(524 \mathrm{mg} / \mathrm{dl}$, nv 165350), ferritin $(612 \mu \mathrm{g} / \mathrm{L}, \mathrm{nv} 30-400)$, procalcitonin $(5.05 \mu \mathrm{g} / \mathrm{L}$, nv $0.02-0.06)$, hypoalbuminemia $(2.7 \mathrm{~g} / \mathrm{dL})$, and hyponatremia $(124 \mathrm{mEq} / \mathrm{L})$. The nasopharyngeal respiratory pathogen testing by 
reverse transcription polymerase chain reaction test (RT-PCR) was positive for Rhinovirus and Enterovirus, and a chest Xray was normal. Intravenous antibiotic therapy with cefotaxime and correction of hyponatremia was started. Given the COVID19 pandemic period, the child was tested for SARS-COV2 with nasopharyngeal swab, negative in two determinations. As the patient experienced worsening abdominal tension and pain, abdominal ultrasound and CT scan were performed with evidence of fluid in pelvis and right iliac fossa, spleen size at the upper limits, and no air-fluid levels. Plain abdominal Xray showed gastrectasia and increased gas in the ileal loops. On day 7 of fever, an empirical intravenous antibiotic therapy with piperacillin/tazobactam and metronidazole was started. Echocardiogram showed normal coronary arteries, minimal pericardial effusion, and mild mitral insufficiency. We were then contacted as reference Center for Kawasaki Disease (KD) in our Pediatric Immunology Unit: considering the clinical history (fever lasting more than 5 days, erythematous rash, labial, and conjunctival hyperemia) and the result of laboratory tests we confirmed the diagnostic suspicion of atypical/incomplete KD without coronary involvement, and started treatment with high dose intravenous immunoglobulins (IVIG) $2 \mathrm{~g} / \mathrm{kg}$ and high dose acetylsalicylic acid (ASA $50 \mathrm{mg} / \mathrm{kg} /$ day). After $36 \mathrm{~h}$ from the end of the IVIG infusion the child was apyretic, but abdominal tension with no bowel movements persisted, and mild respiratory distress with tachypnea and oxygen desaturation (91-92\%) appeared. Chest X-ray, lung ultrasound, and echocardiogram were performed, showing pulmonary infiltrates at the right base and minimal pericardial effusion. Because of the worsening of respiratory and abdominal conditions, he was transferred to our hospital. When admitted, he was tested for SARS-COV2 and the nasopharyngeal aspirate resulted positive. Repeated family history revealed that mother and grandmother had been symptomatic for fever, gastroenteritis, and cough in the previous weeks, and that later the father had experienced rhinitis, fever, cough, and ageusia. Because of mild respiratory distress, the patient needed low-flow oxygen administration for 3 days. A repeated chest X-ray showed only accentuated bronchovascular markings in bilateral peri-hilar and paracardiac regions. Considering the persistence of abdominal pain, no passage of stool or gas for more than $48 \mathrm{~h}$ from admission, a new abdominal $\mathrm{x}$-ray without contrast was performed, highlighting ileocolic meteorism with multiple small diffuse air-fluid levels. Abdominal pain with distension and lack of bowel movements were compatible with paralytic ileus associated with intestinal vasculopathy in KD. Enema and polyethylene glycol (PEG) oral therapy resolved the abdominal symptoms, ASA $5 \mathrm{mg} / \mathrm{kg} / \mathrm{die}$ therapy was started after $48 \mathrm{~h}$ of apyrexia, and laboratory blood tests showed progressive reduction in inflammation markers and rapid normalization of liver enzymes. In the setting of COVID-19 there are several reports of using corticosteroids for Acute Respiratory Disease Syndrome (ARDS), but considering the not severe clinical course and presentation in our patient we did not start steroid therapy. At discharge echocardiogram showed normal coronary arteries, mild mitral insufficiency, and resolution of pericardial effusion. Nasopharyngeal swab and aspirate for SARS-COV-2 resulted positive, so the family was instructed to keep him in quarantine at home, until repetition of SARS-COV-2 test visit and follow-up visit for $\mathrm{KD}$, which are scheduled in the next future.

\section{DISCUSSION}

$\mathrm{KD}$ is an acute, febrile, self-limited systemic vasculitis of small and medium sized vessels and represents the most common cause of acquired heart disease in children in developed countries (6).

Typical or classic KD is characterized by the presence of $\geq 5$ days of fever and $\geq 4$ of the following clinical features: bilateral non-exudative conjunctivitis, erythema of lips and oral mucosa, changes in the extremities, skin rash, and cervical lymphadenopathy with or without coronary artery abnormalities (CAA). Incomplete KD occurs in patients presenting a typical fever without a sufficient number of main clinical criteria, with or without CAA. Atypical KD occurs in patients presenting typical fever and signs or symptoms different from the main clinical features of KD (e.g., meningeal inflammation, seizures, facial paralysis, acute abdomen, acute pancreatitis, cholestatic jaundice, arthritis, renal injury, pneumonia, etc.), with or without CAA $(6,7)$.

The cause of $\mathrm{KD}$ is still unknown but may be related to the combined effects of infections, immune response, and genetic susceptibility. Many studies have reported the role of viral infections in $\mathrm{KD}$ as a possible trigger, including enterovirus, rhinovirus, herpesviruses, adenoviruses, influenza, parainfluenza, and coronaviruses $(8,9)$.

In our case the child had a demonstrated infection by enterovirus, rhinovirus, and SARS-COV-2 according to PCR tests. Many viral infections may elicit systemic inflammation and consequently vasculitides such as $\mathrm{KD}$, and it is also known that SARS-CoV-2 infection can activate uncontrolled inflammation which can lead to local and systemic tissue damage (10). In our patient, more than one pathogen were detected, making it difficult to understand which of the three viral agents had been the trigger.

Our hospital protocol for $\mathrm{KD}$, based on international guidelines $(6,7)$, recommends the troponin $\mathrm{T}$ and BNP dosage only in case of documented myocarditis and/or pericarditis and/or early aneurysms. Since the patient was IVIG responder, electrocardiograms did not reveal signs of ischemia and the echocardiograms performed did not show coronary artery abnormalities or depression of the ejection fraction, we did not measure troponin T or BNP. Moreover, blood tests performed during admission to our hospital, showed a reduction in CRP and normalization of liver enzymes so other investigations would not provide additional information.

Gastrointestinal involvement is not common in $\mathrm{KD}$; the most common gastrointestinal manifestations include vomiting, diarrhea, abdominal pain, hepatomegaly, paralytic ileus, and hydrops of gallbladder $(11,12)$. In many cases of KD with gastrointestinal involvement, the typical signs and symptoms of KD often occur after the intestinal ones (11-13), and the delayed appearance of typical symptoms can be a cause of delayed therapy, which in turn can lead to a greater probability of cardiac 
involvement $(13,14)$. In our case however suggestive symptoms for KD began simultaneously with intestinal symptoms.

Pediatric presentation of SARS-COV-2 infection include completely asymptomatic subjects and patients presenting with mild symptoms like fever, cough, sore throat, vomiting, nausea, and diarrhea (15). Indeed, the clinical course and presentation of COVID-19 in our patient were not severe, even if he transiently needed respiratory support with low-flow oxygen. Considering the mild respiratory presentation, the absence of pathognomonic signs at chest X-ray and the good clinical evolution, we did not start a specific therapy for COVID-19.

To the best of our knowledge, up to now there is only one other published case of KD with concurrent COVID-19, a 6-month-old baby with a complete form of KD (5). However, cases are being informally reported among pediatricians, and recently patients with severe forms including myocarditis and cytokine storm syndromes have been seen in different countries e.g., Italy, France, Netherlands, and UK. An alert has been issued (https://mailchi.mp/05cc3497cda5/alert-possiblesars-cov-2-related-inflammatory-syndrome-in-children?e= 2e634f02aa) emphasizing the apparent rise in the number of children presenting with a multisystem inflammatory state requiring intensive care with overlapping features of toxic shock syndrome and atypical Kawasaki. Interestingly, abdominal pain and gastrointestinal symptoms have been a common feature in these patients, such as in our case. Both COVID-19 infection and $\mathrm{KD}$ are characterized by cytokine release, and both can be treated with anticytokines (anti-IL-1 in particular) in severe

\section{REFERENCES}

1. Lu X, Zhang L, Du H, Zhang J, Li YY, Qu J, et al. SARS-CoV-2 infection in children. N Engl J Med. (2020) 382:1663-5. doi: 10.1056/NEJMc20 05073

2. Livingston E, Bucher K. Coronavirus disease 2019(COVID-19) in Italy. JAMA. (2020) 323:1335. doi: 10.1001/jama.2020.4344

3. $\mathrm{Wu} \mathrm{Z}$, McGoonan JM. Characteristics of and important lessons from the coronavirus disease 2019 (COVID-19) outbreak in China. Summary of a report of 72314 cases from the chinese center for disease control and prevention. JAMA. (2020) 323:1239-42. doi: 10.1001/jama.202 0.2648

4. Harahsheh A, Dahdah N, Newburger J, Portman MA, Piram M, Tulloh $\mathrm{R}$, et al. Missed or delayed diagnosis of Kawasaki disease during the 2019 novel coronavirus disease (COVID-19) pandemic. J Pediatr. (2020). doi: 10.1016/j.jpeds.2020.04.052. [Epub ahead of print].

5. Jones VG, Mills M, Suarez D, Hogan CA, Yeh D, Segal JB, et al. COVID19 and Kawasaki disease: novel virus and novel case. Hosp Pediatr. (2020) 10:537-40. doi: 10.1542/hpeds.2020-0123

6. McCrindle BW, Rowley AH, Newburger JW, Burns JC, Bolger AF, Gewitz $\mathrm{M}$, et al. Diagnosis, treatment, and long-term management of Kawasaki disease, a scientific statement for health professionals from the American heart. Circulation. (2017) 135:927-99. doi: 10.1161/CIR.00000000000 00484

7. Marchesi A, Tarissi de Jacobis I, Rigante D, Rimini A, Malorni W, Corsello G, et al. Kawasaki disease: guidelines of the Italian society of pediatrics, part I - definition, epidemiology, etiopathogenesis, clinical expression and management of the acute phase. Ital J Pediatr. (2018) 44:102. doi: 10.1186/s13052-018-0536-3 cases. A cytokine storm syndrome, similar to macrophage activation syndrome, can in fact appear in both conditions $(16,17)$. The connection between viral infections and $\mathrm{KD}$, the analogies between the severe end of the two conditions, with myocarditis having been reported as well, opens new perspectives with regard to etiopathogenesis. A registry of $\mathrm{KD}$ cases associated with SARS-COV-2 infection is currently ongoing in Italy, and hopefully results will shed light on this unusual association.

\section{DATA AVAILABILITY STATEMENT}

The raw data supporting the conclusions of this article will be made available by the authors, without undue reservation.

\section{ETHICS STATEMENT}

Written informed consent was obtained from next of kin for the publication of any potentially identifiable images or data included in this article.

\section{AUTHOR CONTRIBUTIONS}

$\mathrm{MC}$ wrote the manuscript and designed the topic. RC and RD designed the topic and revised the manuscript. LB revised the manuscript. SG and RP contributed to clinical diagnosis. All authors read and approved the final manuscript and agree to be accountable for the content of the work.

8. Chang LY, Lu CY, Shao PL, Lee PI, Lin MT, Fan TY, et al. Viral infections associated with Kawasaki disease. J Formos Med Assoc. (2014) 113:14854. doi: 10.1016/j.jfma.2013.12.008

9. Turnier JL, Anderson MS, Heizer HR, Jone PN, Glodé MP, Dominguez SR. Concurrent respiratory viruses and Kawasaki disease. Pediatrics. (2015) 136:609-14. doi: 10.1542/peds.2015-0950

10. Cao X. COVID-19: immunopathology and its implications for therapy. Nat Rev Immunol. (2020) 20:269-70. doi: 10.1038/s41577-0200308-3

11. Yaniv L, Jaffe M, Shaoul R. The surgical manifestations of the intestinal tract in Kawasaki disease. J Pediatr Surg. (2005) 40:e1-4. doi: 10.1016/j.jpedsurg.2005.05.063

12. Sun Q, Zhang J, Yang Y. Gallbladder hydrops associated with Kawasaki disease: a case report and literature review. Clin Pediatr. (2018) 57:3413. doi: $10.1177 / 0009922817696468$

13. Colomba C, La Placa S, Saporito L, Corsello G, Ciccia F, Medaglia A, et al. Intestinal involvement in Kawasaki disease. J Pediatr. (2018) 202:18693. doi: 10.1016/j.jpeds.2018.06.034

14. Fabi M, Corinaldesi E, Pierantoni L, Mazzoni E, Landini C, Bigucci B, et al. Gastrointestinal presentation of Kawasaki disease: a red flag for severe disease? PLoS ONE. (2018) 13:e0202658. doi: 10.1371/journal.pone.02 02658

15. Dong Y, Mo X, Hu Y, Qi X, Jiang F, Jiang Z, et al. Epidemiological characteristics of 2143 pediatric patients with 2019 coronavirus disease in China. Pediatrics. (2020). doi: 10.1542/peds.2020-0702

16. García-Pavón S, Yamazaki-Nakashimada MA, Báez M, Borjas-Aguilar KL, Murata C. Kawasaki disease complicated with macrophage activation syndrome: a systematic review. J Pediatr Hematol Oncol. (2017) 39:44551. doi: 10.1097/MPH.0000000000000872 
17. Henderson LA, Canna SW, Schulert GS, Volpi S, Lee PY, Kernan KF, et al. On the alert for cytokine storm: immunopathology in COVID-19. Arthritis Rheumatol. (2020). doi: 10.1002/art.41285. [Epub ahead of print].

Conflict of Interest: The authors declare that the research was conducted in the absence of any commercial or financial relationships that could be construed as a potential conflict of interest.
Copyright (c) 2020 Cazzaniga, Baselli, Cimaz, Guez, Pinzani and Dellepiane. This is an open-access article distributed under the terms of the Creative Commons Attribution License (CC BY). The use, distribution or reproduction in other forums is permitted, provided the original author(s) and the copyright owner(s) are credited and that the original publication in this journal is cited, in accordance with accepted academic practice. No use, distribution or reproduction is permitted which does not comply with these terms. 